\title{
In an expanding universe, what doesn't expand?
}

\author{
Richard H. Price \\ Department of Physics \& Astronomy, Center for Gravitational Wave Astronomy, \\ and Center for Advanced Radio Astronomy, University of Texas at Brownsville, Brownsville, TX 78520 \\ Joseph D. Romano \\ Department of Physics \& Astronomy and Center for Gravitational Wave Astronomy, \\ University of Texas at Brownsville, Brownsville, TX 78520
}

\begin{abstract}
The expansion of the universe is often viewed as a uniform stretching of space that would affect compact objects, atoms and stars, as well as the separation of galaxies. One usually hears that bound systems do not take part in the general expansion, but a much more subtle question is whether bound systems expand partially. In this paper, a definitive answer is given for a very simple system: a classical "atom" bound by electrical attraction. With a mathemical description appropriate for undergraduate physics majors, we show that this bound system either completely follows the cosmological expansion, or — after initial transients - completely ignores it. This "all or nothing" behavior can be understood with techniques of junior-level mechanics. Lastly, the simple description is shown to be a justifiable approximation of the relativistically correct formulation of the problem.
\end{abstract}

\section{INTRODUCTION}

It is not hard to explain to students that the galaxies are moving apart like pennies glued to the surface of an expanding balloon or raisins in an expanding loaf of raisin bread [1 5]. The expanding material represents the uniform stretching of space. But if space itself is stretching, does this mean that everything in it is stretching? Are galaxies growing larger? Are atoms? The usual answer is that "bound" systems do not take part in the cosmological expansion. But if space is stretching how can these systems not be at least slightly affected? And what would it mean for a bound system to be "slightly affected"? Would the bound system, for example, expand at a slower rate? While the universe expands by a factor of $10^{6}$, would a galaxy expand by, say, a factor of $10^{3}$ ? Would less bound systems expand more nearly with the full cosmological rate?

It turns out that these questions get a spectrum of different answers from experts caught unprepared. Part of this confusion is the indeterminacy of just what the question means. (See [ 6] for the effects of cosmological expansion on clusters of galaxies; see [7] and [8] for more mathematical analyses, and for further recent references.) In this article, we will put aside some subtleties, we will focus on a clear simple question, and we will find a clear and interesting answer.

The simple question will take the form of a simple model: a classical "atom," with a negative charge of negligible mass (the "electron") going around a much more massive oppositely charged "nucleus." The Coulomb binding of the atom is physically no different from the gravitational binding of a "small" configuration, like a solar system or a galaxy, but allows certain technical simplifications [9]. We will put this classical atom in a homogeneous universe in which expansion is described by an expansion factor $a(t)$, where $t$ is time. Our goal will be to find the extent to which the growth of $a(t)$ causes the atom to grow, i.e., causes the electron orbit to increase in radius.

In the description of the atom, it will be useful to use two sets of spatial coordinates, both of them spherical polar coordinates with the massive nucleus at the origin. The first system consists of the "physical" coordinates $r, \theta, \phi$ in which $r$ is the proper distance from the nucleus to the electron at a given moment of time. The second set is "cosmological" coordinates $R, \theta, \phi$; a point at fixed values of $R, \theta, \phi$ is a point fixed in the stretching space of the universe and taking part in the cosmological expansion. The two coordinate systems are related by

$$
r=a(t) R .
$$

The angular coordinates $\theta$ and $\phi$ are the same in both the physical and the cosmological coordinates since we can think of the cosmological expansion as proceeding radially outward from the (arbitrarily chosen) origin.

The question whether the atom takes part in the cosmological expansion is then the question: Does the electron follow a trajectory of bounded $r$ (no atomic expansion), or of constant $R$ (full cosmological expansion of the atom), or does the electron do something "in between"?

The nature of the expansion is encoded in the functional form of $a(t)$, and the choice of this function is the choice of the kinematics of the expanding universe. The question of what does or does not expand is a kinematical question fundamentally unrelated to the physics that constrains the form of $a(t)$. For that reason we shall use choices of $a(t)$ 
that lead to the clearest insights, but we will comment on the relationship of these convenient examples to realistic expansion laws.

The answers given by our model contain both expected and unexpected features. An expected feature is that the comparative strengths of the expansion and of the electrical binding determine whether the atom expands. An unexpected feature is the "all or nothing" effect of expansion. We shall see that a sufficiently loosely bound electron will expand with the universe; it will move with constant $R$. A more tightly bound electron will, after some initial disturbance of its orbit, ignore the continuing expansion and maintain bounded $r$; there is no intermediate behavior. We shall also see that this "all or nothing" behavior makes physical sense.

This paper analyzes the expanding atom at two different levels. First, in Sec. II the description uses only Newtonian mechanics and basic electrostatics, and should be accessible to physics students in the junior year. Expansion effects are introduced in this model through a very plausible heuristic stretching force in the relatively simple differential equation for the orbital radius $r(t)$. This model leads to particularly clear graphical insights in the case of cosmological expansion that is exponential in time. Numerical results for this and another model expansion are given to reinforce the "all or nothing" feature of the atomic expansion.

Second, in Sec. III the same classical atom is analyzed using the kinematics of general relativity and Maxwell electrodynamics in curved spacetime[10]. The result of this analysis is a differential equation for $r(t)$ that differs slightly from that in Sec. II] We show, however, that the difference is not significant. If the atom is chosen to be initially nonrelativistic, then subsequent relativistic effects are unimportant. Section IV] summarizes the conclusions of the paper.

\section{NEWTONIAN ANALYSIS}

Our model consists of an unmoving massive nucleus fixed at the origin of a spherical polar coordinate system $r, \theta, \phi$. The position of an electron of mass $m$ orbiting in the equatorial plane $\theta=\pi / 2$ is described by the functions $r(t)$, $\phi(t)$. Since only radial forces act on the electron, its angular momentum $m r^{2} d \phi / d t$ is conserved, and we define the constant of motion

$$
L \equiv r^{2} \frac{d \phi}{d t}
$$

to be the electron angular momentum per unit mass. In the absense of cosmological expansion effects, the equation of motion for $r(t)$ is derived in the usual way, and takes the familiar form

$$
\frac{d^{2} r}{d t^{2}}-\frac{L^{2}}{r^{3}}=-\frac{C}{r^{2}} .
$$

The constant of electrostatic attraction $C$, in SI units, is $Q q /\left(4 \pi \epsilon_{0} m\right)$, where $Q q$ is the magnitude of the product of the nuclear and electron charges.

We need now to consider introducing the effect of expansion. According to Eq. (1), a point fixed in the cosmological expansion, i.e., a point of constant $R, \theta, \phi$, has a radial acceleration[11]

$$
\left.\frac{d^{2} r}{d t^{2}}\right|_{\text {expansion }}=r \frac{d^{2} a / d t^{2}}{a} .
$$

It seems plausible, therefore, that we can treat this term as a radial force per unit mass, and add it to Eq. (3) to arrive at

$$
\frac{d^{2} r}{d t^{2}}-\frac{L^{2}}{r^{3}}=-\frac{C}{r^{2}}+r \frac{d^{2} a / d t^{2}}{a}
$$

From the solution of this equation, and the chosen expansion factor $a(t)$, we can find the radial position $R(t)$ of the electron by using Eq. (1). If we combine $r(t)$ or $R(t)$ with $\phi(t)$ from the integration of Eq. (2), we arrive at a complete description of the orbit in either physical or cosmological coordinates.

At the outset we should notice that the comparative strength of the electrostatic and cosmological terms in Eq. (5) can be usefully cast as a comparison of timescales for atomic and expansion effects. We define the atomic timescale $T_{\text {atom }}$ as a combination of the parameters $L$ and $C$ relevant to the electron's motion

$$
T_{\text {atom }}=L^{3} / C^{2},
$$

and we note that the time for the electron to complete a circular orbit, in the absence of expansion effects, is $2 \pi T_{\text {atom }}$. 
We will first choose the cosmological expansion kinematics to be exponential

$$
a(t)=e^{t / T_{\exp }} .
$$

Such expansion, a "de Sitter" cosmology, is of interest in connection with inflationary models, and mathematical relativity, but it is our first choice for a very different reason: it results in a form of Eq. (5) without any explicit time dependence:

$$
\frac{d^{2} r}{d t^{2}}=\frac{L^{2}}{r^{3}}-\frac{C}{r^{2}}+\frac{r}{T_{\exp }^{2}}
$$

Since $t$ does not explicitly appear, the equation can be viewed as that for a particle moving in one dimension under the influence of an $r$-dependent potential.

This view is based on the fact that Eq. (8) guarantees that the energy-like quantity

$$
E \equiv \frac{1}{2}\left(\frac{d r}{d t}\right)^{2}+\frac{L^{2}}{2 r^{2}}-\frac{C}{r}-\frac{r^{2}}{2 T_{\exp }^{2}}
$$

is constant, so

$$
V \equiv \frac{L^{2}}{2 r^{2}}-\frac{C}{r}-\frac{r^{2}}{2 T_{\exp }^{2}}
$$

can be viewed as an effective potential.

Plots of this potential are given in Fig. 1. Both the potential and the radius are made dimensionless by multiplying them by appropriate combinations of the parameters $L$ and $C$. Each curve is labeled with the value of the parameter $T_{\text {atom }} / T_{\exp }$ that determines the importance of the cosmological expansion to the evolution of the radius of the atom. The larger the value of $T_{\text {atom }} / T_{\text {exp }}$, the larger is the effect of expansion.

Expansion is absent for the top curve, that for which $T_{\text {atom }} / T_{\exp }=0$. In this case, the electron is always trapped in the potential well, i.e., it is permanently bound. If it is started at the bottom of the potential well, at $r=L^{2} / C$ (i.e., at $E=-C^{2} / 2 L^{2}$ in Eq. (9)) it will remain in a circular orbit at that radius. For any larger value of $E$ the electron will orbit in an ellipse.

For nonzero values of the $T_{\text {atom }} / T_{\exp }$ parameter, the potential at large $r$ eventually becomes negative and decreasing; it represents a dominant force outward. Consequently, an electron that is at sufficiently large radius will be driven to even larger radius. The important question is whether the electron will ever get to that region of dominant outward force. The answer is contained in the shapes of the curves in Fig. 1.

We first consider the case in which the electron starts at the bottom of the potential well of the no-expansion curve, then is "surprised" by the sudden turn on of expansion, so that the electron has energy $E=-C^{2} / 2 L^{2}$ and finds itself under the influence of one of the curves with $T_{\text {atom }} / T_{\exp }>0$. For this scenario a critical value of $T_{\text {atom }} / T_{\exp }$ is 0.25 . As shown by the dashed line in Fig. 1 this is the value for which the local peak of the potential has the same value as the lowest point in the potential well of the no-expansion curve. For values of $T_{\text {atom }} / T_{\exp }$ less than 0.25 the surprised electron will remain trapped in an approximately elliptical orbit. For larger values of $T_{\text {atom }} / T_{\exp }$ the electron will move to larger radius and be accelerated outward by the cosmological expansion.

A somewhat different scenario can be envisioned: the electron finds itself in the bottom not of the well of the no-expansion curve, but at the bottom of the potential well of the curve that includes the expansion term. In this case the electron will remain at a fixed value of $r$, the location of the bottom of the potential well, but this can happen only if there actually exists such a potential well. As shown in Fig. 1 there is a critical curve that separates potentials with and without a potential well. That curve turns out to correspond to $T_{\text {atom }} / T_{\exp }=3 \sqrt{3} / 16 \approx 0.3248$.

With the viewpoint of the potentials it is clear why there is an "all or nothing" behavior of the atom. The electron either is, or is not, trapped in the potential well; there is no "partial expansion" possible. Underlying this graphical understanding is a broader but less precise heuristic explanation of the "all or nothing" effect, an explanation that goes beyond expansion that is exponential in time. The cosmological expansion term $r\left(d^{2} a / d t^{2}\right) / a$ increases at large physical distances $r$ from the nucleus. The centrifugal and electrical forces decrease. This implies a sort of instability with respect to expansion. If the electron moves sufficiently far outward, the expansion term will become more important and push the electron yet further outward.

We can get another viewpoint on the bound vs. unbound issue by solving Eq. (8), the equation of motion for the electron, computationally. In principle we could start the computation with the electron at the bottom of a potential well for expansion. The results turn out to be in agreement with the predictions of the analysis based on Fig. 1: the electron stays at a constant value of $r$, so this result is not of particular interest. More interesting is the surprised 


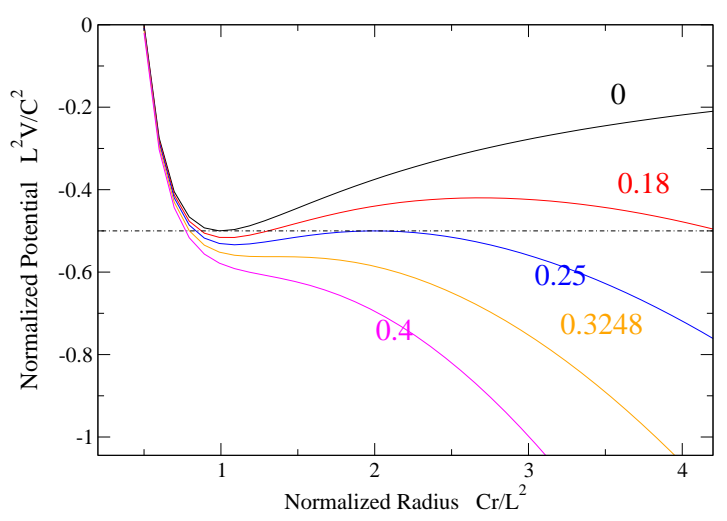

FIG. 1: Effective potential for exponential expansion. Curves are marked by the value of the parameter $T_{\text {atom }} / T_{\text {exp }}$. The curve labeled 0 is the no-expansion potential, the potential for $T_{\text {atom }} / T_{\exp }=0$. The dashed line shows the alignment of the minimum of this no-expansion potential with the local maximum of the potential for $T_{\text {atom }} / T_{\exp }=0.25$.
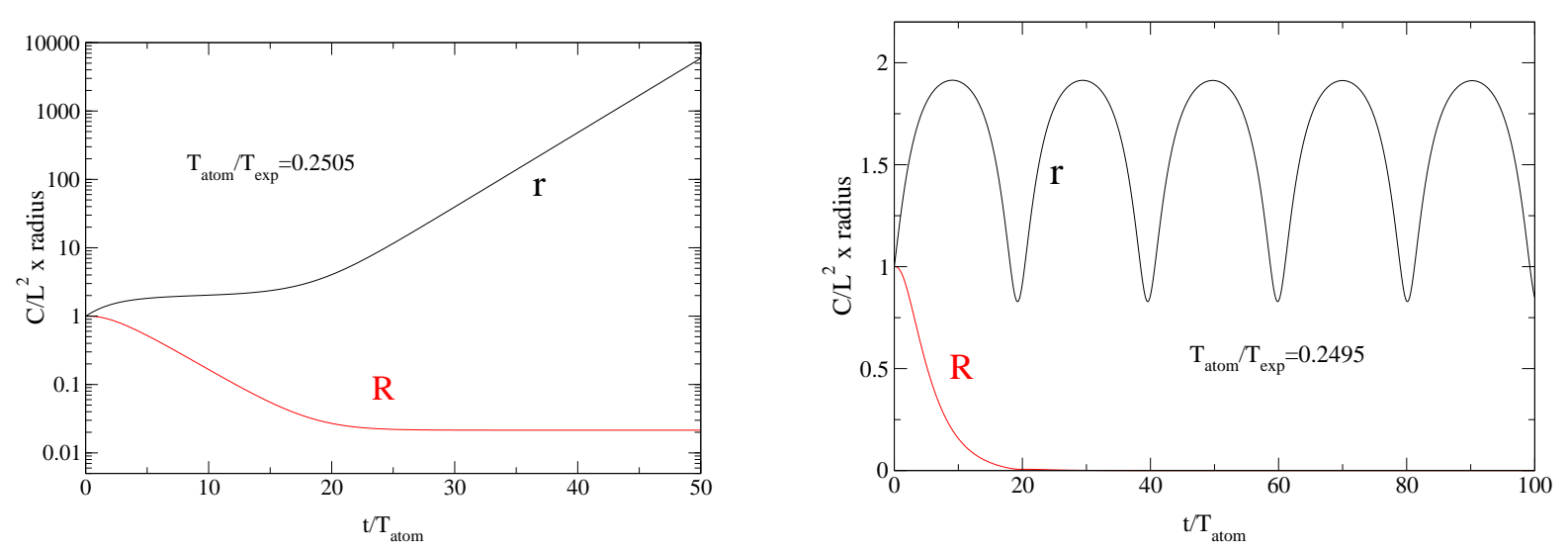

FIG. 2: Radius as a function of time for exponential expansion. On the left is the case for $T_{\text {atom }} / T_{\exp }=0.2505$ for which the electron cosmological radius $R$ remains approximately constant after an initial decrease to about $2 \%$ of its initial value. Due to the exponential increase in $a(t)$, the physical radius $r$ grows without bound. On the right is the radial kinetics for a slighly smaller value, 0.2495 , of $T_{\text {atom }} / T_{\text {exp }}$. In this case the electron remains bound in an approximately elliptical orbit with the physical radius oscillating between values near the original atomic radius. The coordinate radius $R$ in this case falls off exponentially.

electron scenario, the case of an electron, in an expanding universe, with initial $r$ and $d r / d t$ such that the electron's energy, according to Eq. (9), corresponds to the bottom of the no-expansion potential well, i.e., the electron energy is $-C^{2} / 2 L^{2}$. The results, shown in Figs. 2, are in accord with the analysis based on Fig. 1. (The energy corresponds to the dashed horizontal line in Fig. 1.) For $T_{\text {atom }} / T_{\exp }$ slightly greater than the 0.25 critical value, $r$ the physical radius of the atom, grows exponentially after an initial hesitation. By contrast, for $T_{\text {atom }} / T_{\exp }$ slightly less than this critical value the electron remains trapped in an approximately elliptical orbit, and is unaffected by the exponential expansion.

It is important to check that our understanding, based on exponential expansion, applies for other expansion laws. This check will be based on $a(t)$ roughly proportional to $t^{2}$. For convenience we will choose the actual dependence to be

$$
a(t)=1+\left(\frac{t}{T_{\exp }}\right)^{2} \tanh \left(t / T_{\exp }\right) .
$$

For $t$ much larger than $T_{\exp }$ this expansion factor is proportional to $t^{2}$ but its properties at $t=0$ simplify our considerations. Both $d a / d t$ and $d^{2} a / d t^{2}$ vanish at $t=0$, so we can start the expansion with both $d r / d t=0$ and $d R / d t=0$. In addition, the expansion term in Eq. (5) vanishes at $t=0$, so there will be no initial cosmological acceleration; if we choose the condition $r=L^{2} / C$ for balance of Coulomb and centripetal force, then the there will be no initial acceleration. 

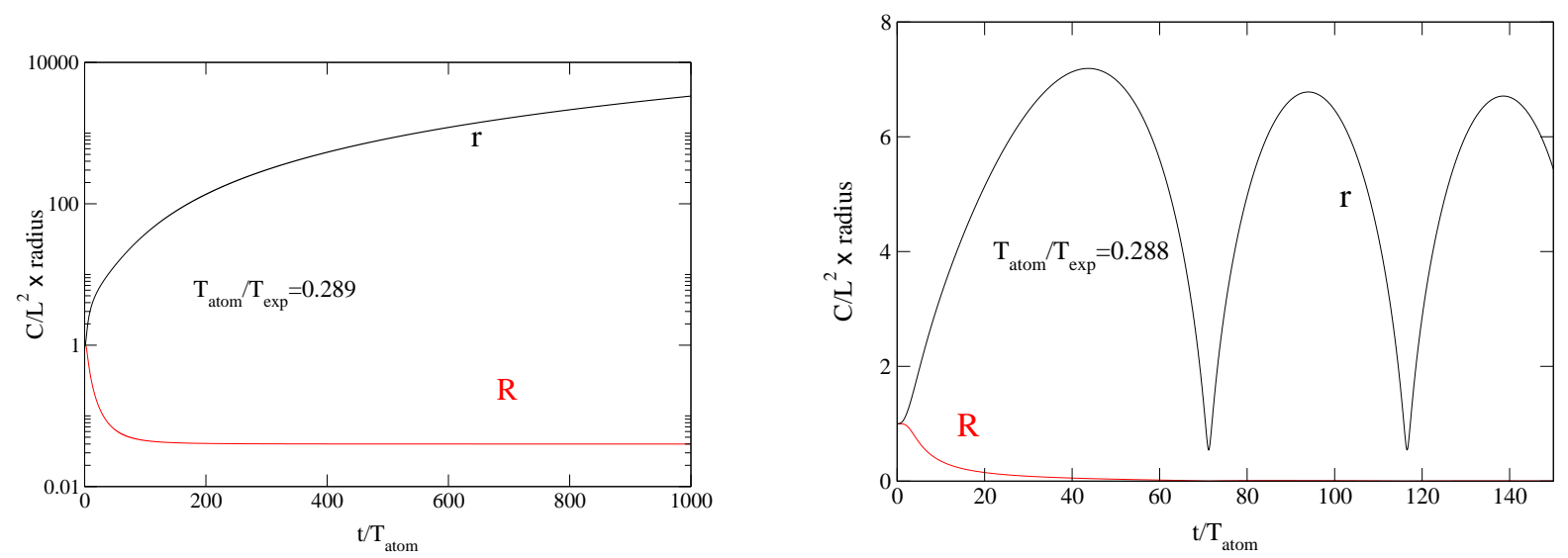

FIG. 3: Radius as a function of time for the modified $a \propto t^{2}$ expansion described in the text. The figure on the left is for $T_{\text {atom }} / T_{\exp }=0.289$ for which the electron is unbound. In this case the cosmological radius $R$ remains constant for large times at about $4 \%$ of its initial value, while the physical radius $r$ expands proportional to $t^{2}$. On the right is shown the radii as a function of time for $T_{\text {atom }} / T_{\exp }=0.288$. Here the cosmological radius $R$ decreases asymptotically to zero roughly as $t^{-2}$, while the physical radius oscillates as the electron orbits in a bound, approximately elliptical, orbit.

Results are shown in Fig. 3 for two very nearby values of the parameter $T_{\text {atom }} / T_{\text {exp }}$. For the expansion law of Eq. (11) we see the same qualitative phenomenon as for the exponential expansion: the atom either fully takes part in the cosmological expansion or, for a slightly smaller value of $T_{\text {atom }} / T_{\exp }$, it remains bound.

\section{RELATIVISTIC ANALYSIS}

The analysis in the previous section was based on a heuristic term in Eq. (5) representing the effect of expansion. Here we analyze the problem using relativisitic cosmology and Maxwell-Einstein theory.

We start with a standard form [12] for the spacetime metric of a homogeneous isotropic universe

$$
d s^{2}=-c^{2} d t^{2}+a^{2}(t)\left[\frac{d R^{2}}{1-k R^{2}}+R^{2}\left(d \theta^{2}+\sin ^{2} \theta d \phi^{2}\right)\right] .
$$

Here, as in Sec. III $a(t)$ is the expansion factor and $R$ is the cosmological radius, with $r=a(t) R$ the physical radius. As we shall explain in more detail below, the presence of the speed of light, $c$, in the line element introduces an additional parameter for relativistic motion, which, for our classical atom, is the ratio of the initial orbital speed of the electron to the speed of light.

The constant $k$ can be positive, negative, or zero, and has a magnitude of order $1 / R_{c}^{2}$, where $R_{c}$ is a characteristic cosmological distance. If $R^{2} / R_{c}^{2}$ is not negligibly small, it means that our atom occupies a significant fraction of the universe. For our considerations of "what expands" we want our atom to be very small compared to the size of the universe, so we omit the $k R^{2}$ term in Eq. (12), i.e., we set $k=0$.

The first step in the relativistic analysis is to find the correct description of the electrical attraction. For the spherically symmetric electromagnetic field of the nucleus there can only be a component $F^{0 R}$ of the electromagnetic tensor $F^{\mu \nu}$. The Maxwell equations $F_{; \beta}^{\alpha \beta}=0$, with $\alpha=0$ and with $\alpha=R$ give us

$$
\frac{1}{R^{2}}\left(F^{0 R} R^{2}\right)_{, R}=0=\frac{1}{a^{3}(t)}\left(F^{0 R} a^{3}(t)\right)_{, t}
$$

so that the solution must have the form

$$
F^{0 R}=\frac{Q}{R^{2} a^{3}} .
$$

The $R$ equation of motion of the electron's 4 -velocity $U^{\alpha}$ is

$$
U^{\alpha} U_{; \alpha}^{R}=\frac{q}{m} U_{0} F^{0 R},
$$


where $q$ is the magnitude of the charge of the electron. For motion in the $\theta=\pi / 2$ plane this becomes, after some manipulations,

$$
\frac{d}{d t}\left(a^{2} \frac{U^{0}}{c} \frac{d R}{d t}\right)-\frac{L^{2}}{a^{2} R^{3}\left(U^{0} / c\right)}=-\frac{C}{a R^{2}} .
$$

Here $L \equiv U_{\phi}=r^{2}\left(U^{0} / c\right) d \phi / d t$ is a constant of the motion, and we have chosen the constant $C$ to be analogous to the same symbol in Eq. (5). We now note that Eq. (5), with $r=a(t) R$, can be written in the form

$$
\frac{d}{d t}\left(a^{2} \frac{d R}{d t}\right)-\frac{L^{2}}{a^{2} R^{3}}=-\frac{C}{a R^{2}} .
$$

The adequacy of the Newtonian analysis therefore depends on the extend to which $U^{0} / c$ differs from unity.

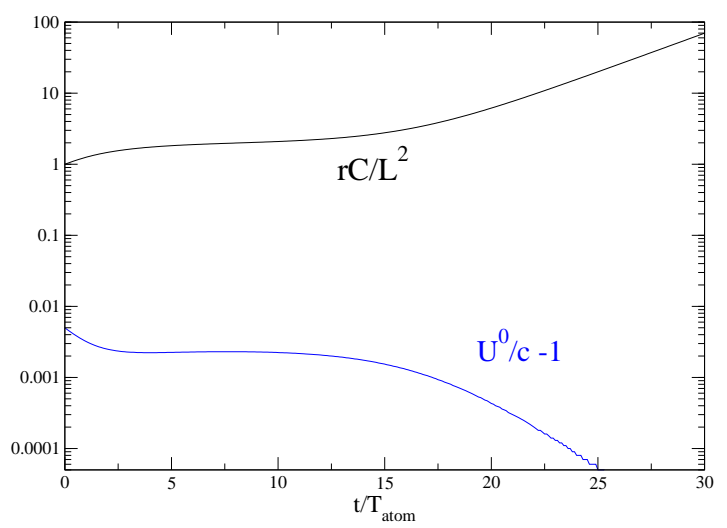

FIG. 4: The unbound physical radius and the index of relativistic effects $U^{0} / c-1$ for exponential expansion with $T_{\text {atom }} / T_{\exp }=$ 0.252 , starting with $d R / d t=0$ and $\beta_{0}=0.1$.

To compute motion for a relativistic model, Eq. (16) must be solved simultaneously with an expression for $U^{0} / c$. This additional expression can be obtained from the normalization of the 4-velocity, $U^{\mu} U_{\mu}=-c^{2}$, leading to

$$
\frac{U^{0}}{c}=\left[1-\left(\frac{a}{c} \frac{d R}{d t}\right)^{2}-\left(\frac{a R}{c} \frac{d \phi}{d t}\right)^{2}\right]^{-1 / 2}
$$

An implicit expression for $U^{0} / c$, which is useful for understanding the subsequent time-evolution of $U^{0} / c$, is

$$
\frac{U^{0}}{c}=\sqrt{1+\left(\frac{a}{c} \frac{U^{0}}{c} \frac{d R}{d t}\right)^{2}+\left(\frac{L}{a R c}\right)^{2}} .
$$

In the Newtonian case a model for the classical atom required only the choice of the expansion law, and a value of a single dimensionless parameter $T_{\text {atom }} / T_{\text {exp }}$. For relativistic motion there is an important difference; we must now choose a second dimensionless parameter $\beta_{0}=v_{0} / c$, where $v_{0}=C / L$ is the initial orbital speed of the electron times $2 \pi$. This need for a second parameter is instructive. If we were to fix, say, $T_{\text {atom }}=T_{\exp }$, this could correspond to slow electron motion (compared to $c$ ) and slow expansion, or to fast electron motion and fast expansion. In the second case, but not the first, relativistic effects would be important.

If $\beta_{0}$ is not chosen small compared to unity, then relativistic effects will be important even initially. Such effects, while interesting in their own right, are not related to cosmological expansion, and are not our focus here. Rather, what is of primary interest is the question whether an atom that is not initially relativistic can become relativistic when it is cosmologically expanding. We investigate this first numerically, with a universe following the exponenetial expansion in Eq. (7). We start with $d R / d t=0$, and choose $\beta_{0}=0.1$ so that the electron starts out mildly relativistic and we can follow the evolution of its relativistic effects. Since we want the atom to be unbounded, we take $T_{\text {atom }} / T_{\exp }$ to be 0.252 . (It turns out that with $\beta_{0} \neq 0$ the unbound behavior requires a slightly larger value of $T_{\text {atom }} / T_{\exp }$ than in the Newtonian case.)

Results for this model are shown in Fig. 4. The plots show the unbounded growth of the physical radius $r$, and show that $U^{0} / c-1$, the measure of the relativistic nature of the electron motion, decreases with the expansion of 
the atom. The mathematical basis for this is not hard to see in Eq. (19). At large expansion, Eq. (16) tells us that the combination $a^{2}\left(U^{0} / c\right) d R / d t$ is approximately constant. This means that the middle term inside the square root of Eq. (19) must fall off as $a^{-2}$. The last term in the square root also falls off with the expanison. The implication, validated by Fig. 4 is that $U^{0} / c-1 \rightarrow 0$ with unbounded expansion.

The mathematical "how" is then clear, but the physical "why" must be explained. In this connection it is interesting to consider the velocity of an unbound electron relative to the "fabric of the universe," i.e., the velocity $v_{\text {loc }}$ that would be measured in the local Minkowski frame of an observer comoving with the cosmological flow[15], an observer with constant $R, \theta$, and $\phi$. That velocity is easily shown to be

$$
v_{\mathrm{loc}}=c \sqrt{1-\left(c / U^{0}\right)^{2}}
$$

As the expansion proceeds, the particle is, in some sense, becoming less and less relativistic.

\section{CONCLUSION}

We have presented a simple definitive question about the influence of the expansion of the universe on a very particular system: a classical "atom." And we have found a simple definitive answer: Expansion forces increase with increasing atomic radius, while atomic forces decrease. This amounts to an instability with respect to the disruption of an atom. If the atomic accelerations are initially stronger than the cosmological, then the subsequent expansion will become less and less important. The atom will not "partially" take part in the expansion. If, on the other hand, the cosmological effect is initially stronger, the atomic radius will increase and the atomic forces will become less and less important. The atom will fully take part in the expansion.

In analyzing this problem we have relied on a simple description of expansion, that of Eq. (5), that avoids relativistic effects. A major pedagogical point is the simple graphical way in which the "what expands" question can be graphically understood for the special case of exponential cosmological expansion.

With an a priori correct general relativistic calculation, and numerics, we have shown that the simple nonrelativistic model is fully adequate. We have also shown that for an atom that is not bound, but that expands with the universe, relativistic effects become less important as the atom gets larger.

We end with a "practical" consideration. Our quantification of the relative strengths of atomic and expansion forces was given in terms of a characteristic time $T_{\text {atom }}$ for the motion of electrons in atoms, and $T_{\exp }$, the cosmological expansion time, e.g., the Hubble time. Our analyses showed that atomic forces are initially stronger if $T_{\text {atom }} / T_{\text {exp }}$ is less than order unity. Since $T_{\text {atom }}$ is around $10^{-16} \mathrm{sec}$., and $T_{\text {exp }}$ is around $4 \times 10^{17}$ sec., atoms are in no danger of being disrupted by cosmological expansion.

\section{ACKNOWLEDGMENT}

This paper is the result of a question posed by high school student Deepak Ramchand Mahbubani, Jr. at UTB's " 21 st Century Astronomy Ambassador's Program," and by the lack of a clear answer at the right level. JDR acknowledges support from NSF grants CREST-0734800 and PHY-0855371. We thank Prof. Bonnor for bringing to our attention Ref. [10].

[1] L. W. Fredrick and R. H. Baker, Astronomy $10^{\text {th }}$ ed. (D. Van Nostrand, New York, 1976), p. 510.

[2] D. Goldsmith, The Evolving Universe (Benjamin/Cummings M enlo Park, 1986), pp. 106-110.

[3] M. Riordan and D. N. Schramm, The Shadows of Creation (Freeman, New York, 1991), p. 16.

[4] R. H. Price and E. Grover, "Cosmological expansion in the classroom," Am. J. Phys. 69, 125-128 (2001).

[5] The analogy of pennies glued to a balloon can be found, for example, in Sec. 27.5 of C. W. Misner, K. S. Thorne, and J. A. Wheeler, Gravitation (W. H. Freeman, San Francisco, 1973).

[6] P. D. Noerdlinger and V. Petrosian, "The Effect of Cosmological Expansion on Self-Gravitating Ensembles of Particles," Astrophysical J. 168, 1 (1971).

[7] J. L. Anderson, "Multiparticle Dynamics in an Expanding Universe," Phys. Rev. Lett. 75, 3602-3604 (1995).

[8] F. I. Cooperstock, V. Faraoni, and D. N. Volleck, "The Influence of the Cosmological Expansion on Local Systems," Astrophysical J. 503, 61 (1998).

[9] Indeed, there are no simplifications needed in the Newtonian approximation. For the relativistic case, however, a separation of the cosmological and local gravitational effects is necessary for a simple analysis. 
[10] W.B. Bonnor, "Size of a hydrogen atom in the expanding universe," Classical and Quantum Grav., 16, 1313 (1999). Our analysis, and results, are in complete agreement with those in this reference, but we focus on the pedagogical aspects of this model.

[11] This heuristic Newtonian result agrees with Eq. (2.8) of the general relativistic analysis in Ref. [8] for the acceleration in Fermi normal coordinates induced by uniform expansion. Note that the analysis in that reference is a valid approximation only for distances from the nucleus small compared to a characteristic cosmological distance. As shown in Sec. III our Eq. (5) applies without such a constraint.

[12] Reference 5], Chap. 27.

[13] S. Perlmutter, "Supernovae, Dark Energy and the Accelerating Universe" Physics Today, April 2003, pp. 53-60.

[14] P. J. E. Peebles and B. Ratra, "The cosmological constant and dark energy," Rev. Mod. Phys. 75, 559-606 (2003).

[15] This viewpoint has been stressed by E. F. Bunn and D. W. Hogg, "The kinematic origin of the cosmological redshift," Am. J. Ph., 77, 688-694 (2009), and is closely related to the analysis in Ref. [8]. 